\title{
Utilization of Obstetric Analgesia for Labor Pain Management and Associated Factors among Obstetric Care Providers in Public Hospitals of Addis Ababa, Ethiopia: A Cross-Sectional Study
}

\author{
Rediet Gido DD, $^{1}$ Tesfaye Assebe Yadeta, ${ }^{2}$ and Abera Kenay Tura ${ }^{2,3}$ \\ ${ }^{1}$ Department of Midwifery, College of Health Science and Medicine, Dilla University, Dilla, Ethiopia \\ ${ }^{2}$ School of Nursing and Midwifery, College of Health and Medical Sciences, Haramaya University, Harar, Ethiopia \\ ${ }^{3}$ Department of Obstetrics and Gynaecology, University Medical Centre Groningen, University of Groningen, \\ Groningen, Netherlands
}

Correspondence should be addressed to Rediet Gido; redigido79@gmail.com

Received 18 March 2021; Revised 28 July 2021; Accepted 30 October 2021; Published 22 November 2021

Academic Editor: Yoshitsugu Chigusa

Copyright (C) 2021 Rediet Gido et al. This is an open access article distributed under the Creative Commons Attribution License, which permits unrestricted use, distribution, and reproduction in any medium, provided the original work is properly cited.

\begin{abstract}
Background. In low-income countries, pain-free labor initiative is an emerging concept and still parturient undergoes through painful labor; this is not different in Ethiopia; despite the national direction to use analgesia for labor pain and strong demand from the women, evidence on utilization of obstetric analgesia for labor pain management in Ethiopia is scarce. The objective of this study was to assess level of obstetric analgesia utilization and associated factors among obstetric care providers in public hospitals in Addis Ababa, Ethiopia. Methods. An institution-based cross-sectional study was used. All obstetric care providers working in labor and delivery units in public hospitals in Addis Ababa were included. The data were collected using a selfadministered structured questionnaire. After checking for completeness, data were entered into Epi-data 3.1 and analyzed using SPSS 20. Bivariate and multivariable logistic regressions were used to identify factors associated with utilization of obstetric analgesia. Result. Of 391 obstetric care providers included in the study, 143 (36.6\%; 95\% CI: 31.5-40.9\%) reported providing labor analgesia. Having adequate knowledge (AOR 2.7; 95\% CI: 1.37-5.23), ten and more years of work experience (AOR 4.3; 95\% CI: 1.81-10.13), and availability of analgesics (AOR 3.3; 95\% CI: 1.99-5.53) were significantly associated with providing labor analgesia. Conclusion. Slightly more than 3 in 10 obstetric care providers reported providing labor analgesics to women. Training of providers and ensuring adequate supply of analgesics is required to make sure that women in labor would not suffer from labor pain.
\end{abstract}

\section{Background}

Although labor is a natural physiologic phenomenon, it is accompanied with severe pain which requires management [1]. Labor pain management with use of pharmacological agents or nonpharmacologic means has become an integral component of labor management [2]. Pain management in labor involves a series of activities that include pain assessment as well as pain control or abolition. Thus, obstetric care providers have a duty to utilize safe and optimal pharmacological interventions in attaining painless labor [3].

Women who are offered analgesics had greater birth satisfaction and healthy reproductive outcomes by addressing both the emotional and physical aspects [4].
However, inadequate labor pain management negatively impacts maternal and fetal wellbeing, as well as progress of labor [5]. It may further lead mothers to postpartum depression, posttraumatic stress disorder, negative experience and dissatisfaction after childbirth, and fear of childbirth, which increases maternal request for cesarean section [6-9].

In high-income countries, pain relief is part of routine labor management and more than $70 \%$ of women got obstetric analgesia $[10,11]$. However, in several low-income countries, including Ethiopia, pain-free labor initiatives are new and level of its utilization remained largely unexplored [12]. The Ethiopian Food, Medicine, and Health Care Administration and Control Authority (FMHACA) recommended healthcare providers to give analgesics and 
anesthetics for labor management [13]. However, level of its utilization among obstetric providers is not yet well explored.

Although lack of analgesic drugs, healthcare delivery systems or knowledge, attitude, and skills of providers may affect utilization of labor analgesia $[14,15]$. Given the national direction to use analgesia for labor pain and considering relief of labor pain as right of laboring women, assessment of provision of labor analgesia among obstetric providers is essential. This study was conducted to assess utilization of obstetric analgesia among obstetric care providers in major public hospitals in Addis Ababa, Ethiopia.

\section{Materials and Methods}

2.1. Study Setting. This is an institution-based cross-sectional study; the structured questioner-based study was conducted in labor and delivery units in public hospitals in Addis Ababa, the capital city of Ethiopia, located at $9^{\circ} 1048^{\prime \prime}$ North and $38^{\circ} 44024^{\prime \prime}$ East with a total land area of 54,000 hectare, divided into 10 subcities and 100 kebeles (lowest administrative units in Ethiopia). During the study period, there were 54 hospitals: 13 governmental, 39 private, and 2 nongovernment organizations hospitals. The study was conducted from February 25 to March 242020 in 10 public hospitals, where more than 7500 healthcare providers were working.

2.2. Study Population. Four hundred twenty-seven obstetric care providers working only in labor and delivery unit were invited, and a written informed consent was obtained after clarification of the purpose of the study, the guidelines to complete the questionnaire, ensuring confidentiality of the responses, and the right to withdraw the results at any time for no reason. Out of the 427 obstetric care providers approached, 391 of them returned completed questionnaires.

2.3. Sample Size and Sampling Procedure. The minimum sample size required for the study was estimated using the single population formula with the following assumptions: utilization of pharmacologic analgesics (45.2\%) [16], 95\% CI, and margin of error of $5 \%$. The calculated sample size was 416. But, considering the overall small number of obstetric care providers working in labor and delivery units $(N=427)$, we included all eligible providers in the selected hospitals.

2.4. Data Collection Method. A structured, self-administrated questionnaire was conducted individually. Written consent was asked for voluntary participation. Contact details of the principal investigator was provided in case a participant had questions or concerns that arose during the study period.

Tools used in the study were adapted from related literatures $[16,17]$. The questionnaire consisted of four parts: sociodemographic conditions, perception about labor pain, institutional-related questions, and use of labor analgesics. The questionnaire was pretested on 20 obstetric care providers working in Bishoftu General Hospital to measure the content validity of the tools in determining the clarity of questions, the effectiveness of the instructions, the completeness of response sets, the time required to complete the questionnaire, and the success of the data collection technique; results obtained were useful in appraisal and modification of the tools; these subjects were not from the study sample.

2.5. Data Processing and Analysis. Data consistency was checked and entered into Epi-data version 3.1, was exported to SPSS version 20 for further analysis, and was summarized by using descriptive statistics. Tables were used for data presentation. Bivariate logistic regression was used to identify factors associated with utilization of obstetric analgesia among obstetric care providers based on adjusted odds ratio (AOR), 95\% CI, and $p$ value $<0.25$.

We used the enter approach in for inclusion into the multivariate model, while the Hosmer-Lemeshow statistic was used for model diagnostics. The multivariable logistic regression model was used to control the possible effect of confounders, and finally, the variables that had independent association with utilization of obstetric analgesia were identified on the basis of AOR, with 95\% CI and $p$ value $\leq 0.05$. The variables were entered to the multivariate model using the backward logistic regression.

\subsection{Operational Definition}

2.6.1. Utilization of Labor Analgesia. Determined as a selfreport of using labor analgesics to manage labor pain in the last delivery they attend [18].

2.6.2. Knowledge. Knowledge-related question comprised of 9 items, and the score ranges from 0 to 9; knowledge was considered as adequate when obstetric care providers scores equal or above mean (i.e., $\geq 5.1$ ) and considered as inadequate when they score below the mean [17].

2.6.3. Attitude. Attitude-related question comprises 7 items, and possible response levels of all items were 5 (strongly disagree, disagree, uncertain, agree, and strongly agree), and total score ranges from 7 to 35 . Then, attitude was considered as positive when obstetric care providers scores equal or above mean (i.e., $\geq 18.8$ ) and considered as negative when they score below the mean after arranging all statements in affirmative way [17].

2.7. Ethical Consideration. This study was conducted in accordance with the Helsinki Declaration [19]. The study protocol was reviewed and approved by the Institutional Health Research Ethics Review Committee (Ref no.: IHRERC 017/2020) of College of Health and Medical Sciences, Haramaya University, Ethiopia. Permission to conduct the study in each hospital was obtained from Addis Ababa Health Bureau and respective hospital heads. Informed, voluntary, and signed consent was obtained from all study participants. 
Confidentiality of participants was maintained through using codes and anonymous questionnaire.

\section{Results}

3.1. Sociodemographic Characteristics. Of 427 obstetric care providers invited to the study, 391 (91.6\%) returned the completed questionnaire and were included in the analysis. The mean age of the respondents was $28.7( \pm 3.8)$ years, ranging from 20 to 48 . Majority of the respondents were 20-29 years old (66\%), midwives (65\%), and worked less than six years $(61.4 \%)$. Almost half of the obstetric care providers are BSC holders (54.5\%), work in specialized/ referral hospitals (52.7\%), and males (51.9\%) (Table 1).

3.2. Utilization of Obstetric Analgesia. A total of 143 (36.6\% (95\% CI: 31.5-40.9\%)) of obstetric care providers reported using labor analgesics to manage labor pain. Only $4.1 \%$ reported using routinely for labor management, while $21.5 \%$ and $11 \%$ reported using sometimes and up on maternal request, respectively. The most commonly used analgesics were pethidine $69(48.3 \%)$, tramadol 60 (42\%), and diclofenac 45 (31.5\%) (Table 2).

3.3. Knowledge and Attitude toward Obstetric Analgesia. The most known pharmacologic method for managing labor pain were systemic opioids $(181 ; 46.3 \%)$, nonopioid systemic analgesia $(169 ; 43.2 \%)$, regional analgesia $(129 ; 33 \%)$, inhalational $(72 ; 18.4 \%)$, and cervical $(53 ; 13.6 \%)$. Among the respondents, $36.1 \%$ asked laboring woman to provide pain relief. Similarly, 36.3\% reported that pharmacologic labor pain management is the best method to manage labor pain. With regard to labor pain, $55.8 \%, 29.2 \%$, and $15.1 \%$ described it as moderate, severe, and mild, respectively. Only $37.9 \%$ of the respondents reported to hear about the WHO pain ladder. In general, only $26.1 \%$ of the respondents had adequate knowledge about labor analgesics (Table 3 ).

Regarding attitude of obstetric care providers, 212 (54.2\%) respondents believed that obstetric analgesia should be given for laboring mothers. In addition, 195 (49.9\%) believe that labor pain of every mother should be managed. Two-thirds $(67.5 \%)$ of the respondents stated that women should not endure labor pain. Although $60.4 \%$ believed that labor analgesia offers better birth experience and $58.6 \%$ had positive attitude toward obstetric analgesia, 36.6\%, 37.1\%, and $43.5 \%$ believe that it affects progress of labor, causes fetal distress, and late presentation, respectively (Table 4).

The main institutional-related reasons that impede utilization of analgesics in labor pain management routinely or at all were reported to be lack of training (231; 59.1\%), absence of guidelines and protocols $(226 ; 57.8 \%)$, lack of skilled obstetric care providers $(221 ; 56.3 \%)$, unavailability of drugs $(193 ; 49.4 \%)$, and cost of analgesics $(83 ; 41.9 \%)$.

3.4. Factors Associated with Utilization of Obstetric Analgesia. Knowledge of labor analgesics, work experience, and availability of analgesics were found to be independently
TABLE 1: Sociodemographic characteristics of obstetric care providers working in labor and delivery in major public hospitals in Addis Ababa, Ethiopia, $2020(n=391)$.

\begin{tabular}{|c|c|c|c|}
\hline \multicolumn{2}{|r|}{ Variables } & $n$ & $\%$ \\
\hline \multirow{3}{*}{ Age } & $20-29$ & 258 & 66 \\
\hline & $30-39$ & 117 & 29.9 \\
\hline & $\geq 40$ & 16 & 4.1 \\
\hline \multirow{2}{*}{ Sex } & Male & 203 & 51.9 \\
\hline & Female & 188 & 48.1 \\
\hline \multirow{4}{*}{ Religion } & Orthodox & 224 & 57.3 \\
\hline & Muslim & 90 & 23 \\
\hline & Protestant & 71 & 18.2 \\
\hline & Catholic & 6 & 15 \\
\hline \multirow{4}{*}{ Profession } & Medical doctor & 95 & 24.3 \\
\hline & Midwife & 254 & 65 \\
\hline & Anesthetist & 32 & 8.2 \\
\hline & Others* & 10 & 2.5 \\
\hline \multirow{5}{*}{ Qualification } & Diploma & 24 & 6.1 \\
\hline & $\mathrm{BSc}$ & 213 & 54.5 \\
\hline & MSc & 53 & 13.6 \\
\hline & General practitioner & 69 & 17.6 \\
\hline & Obgyn (including residents) & 32 & 8.2 \\
\hline \multirow{3}{*}{ Work experience } & $\leq 5$ & 240 & 61.4 \\
\hline & $6-9$ & 93 & 23.8 \\
\hline & $\geq 10$ & 58 & 14.8 \\
\hline \multirow{2}{*}{ Level of hospital } & General hospital & 185 & 47.3 \\
\hline & Specialized/referral & 206 & 52.7 \\
\hline
\end{tabular}

Obgyn, obstetrician and gynecologist. Others*, nurses and anesthesiologists.

TABLE 2: Types of labor analgesia offered by obstetric care providers in public hospitals of Addis Ababa, Ethiopia, $2020(n=143)$.

\begin{tabular}{lccc}
\hline \multicolumn{2}{c}{ Types of labor analgesics offered } & $n$ & $\%$ \\
\hline \multirow{2}{*}{ Systemic opioids } & Pethidine & 69 & 48.3 \\
& Tramadol & 60 & 42 \\
\hline \multirow{2}{*}{ Systemic nonopioids } & Diclofenac & 45 & 31.5 \\
& Paracetamol & 24 & 16.8 \\
& Hyoscine & 8 & 5.6 \\
\hline Regional analgesic & Epidural analgesic & 18 & 12.6 \\
\hline
\end{tabular}

associated with utilization of obstetric analgesia. Providers with $\geq 10$ year work experience were 4.3 (AOR: 4.3 ; 95\% CI: 1.81-10.13) times more likely to use obstetric analgesia compared to those who practiced $\leq 5$ years. Obstetric providers with adequate knowledge were 2.7 (AOR: $2.7 ; 95 \% \mathrm{CI}$ : 1.37-5.23) times more likely to use obstetric analgesics compared to their counterparts. In addition, healthcare providers working in facilities where analgesics were readily accessible were 3.3 (AOR: 3.3; 95\% CI: 1.99-5.53) times more likely to use labor analgesia compared to providers working in hospitals where analgesics were not readily available (Table 5).

\section{Discussion}

This study was conducted to assess utilization obstetric analgesia for labor pain management among obstetric care providers in public hospitals in Addis Ababa, Ethiopia. We found that almost 4 in $10(36.6 \%)$ providers reported 
TABle 3: Knowledge on obstetric analgesia among obstetric care providers in public hospitals of Addis Ababa, Ethiopia, 2020 ( $n=391$ ).

\begin{tabular}{|c|c|c|c|}
\hline \multicolumn{2}{|l|}{ Response } & $n$ & $\%$ \\
\hline \multirow{2}{*}{ Know labor analgesics } & Yes & 242 & 61.9 \\
\hline & No & 149 & 38.1 \\
\hline \multirow{5}{*}{ Analgesics methods } & Systemic opioids & 181 & 46.3 \\
\hline & Systemic nonopioids & 169 & 43.2 \\
\hline & Regional analgesics & 129 & 33 \\
\hline & Inhalational & 72 & 18.4 \\
\hline & Cervical & 53 & 13.6 \\
\hline \multirow{2}{*}{ Ever asked to provide labor pain analgesics } & Yes & 141 & 36.1 \\
\hline & No & 250 & 63.9 \\
\hline \multirow{4}{*}{ Best method for managing labor pain } & Nonpharmacologic & 127 & 32.5 \\
\hline & Pharmacologic & 142 & 36.3 \\
\hline & Slapping & 15 & 3.8 \\
\hline & Nothing & 120 & 30.7 \\
\hline \multirow{2}{*}{ Heard about WHO pain ladder } & Yes & 148 & 37.9 \\
\hline & No & 243 & 62.1 \\
\hline \multirow{2}{*}{ Adequate knowledge } & Yes & $102(26.1)$ & \\
\hline & No & $289(73.90)$ & \\
\hline
\end{tabular}

TABle 4: Attitude toward obstetric analgesia among obstetric care providers in public hospitals of Addis Ababa, Ethiopia, 2020 ( $n=391$ ).

\begin{tabular}{|c|c|c|c|}
\hline Question & Disagree, $n(\%)$ & Neutral, $n(\%)$ & Agree, $n(\%)$ \\
\hline Obstetric analgesia should be given for labor pain management & $176(45)$ & $3(0.8)$ & $212(54.2 \%)$ \\
\hline Every laboring women should be managed with analgesics & $174(44.5)$ & $22(5.6)$ & $195(49.9)$ \\
\hline Women should endure labor pain & $264(67.5)$ & $44(11.3)$ & $83(21.2)$ \\
\hline Labor analgesia influences labor progress & $207(52.9)$ & $41(10.5)$ & $143(36.6)$ \\
\hline Labor analgesia causes late presentation & $171(43.7)$ & $50(12.8)$ & $170(43.5)$ \\
\hline Labor analgesia causes fetal distress & $208(53.2)$ & $38(9.7)$ & $145(37.1)$ \\
\hline Labor analgesia offers a better birth experience & $137(35.1)$ & $18(4.6)$ & $236(60.4)$ \\
\hline Overall attitude level & \multicolumn{2}{|c|}{ Positive } & $229(58.6)$ \\
\hline & \multicolumn{2}{|c|}{ Negative } & $162(41.4)$ \\
\hline
\end{tabular}

TABLE 5: Factors associated with utilization of obstetric analgesia among obstetric care providers in public hospitals of Addis Ababa, Ethiopia, 2020.

\begin{tabular}{|c|c|c|c|c|c|}
\hline \multicolumn{2}{|c|}{ Variables } & \multicolumn{2}{|c|}{ Utilized } & \multirow{2}{*}{ COR $(95 \% \mathrm{CI})$} & \multirow{2}{*}{ AOR $(95 \% \mathrm{CI})$} \\
\hline$v a_{11}$ & & Yes & No & & \\
\hline \multirow{3}{*}{ Work experience } & $\leq 5$ & 73 & 167 & 1 & 1 \\
\hline & $6-9$ & 32 & 61 & $1.2(0.72-1.99)$ & $1.25(0.63-2.50)$ \\
\hline & $\geq 10$ & 38 & 20 & $4.35(2.37-7.98)$ & $4.3(1.81-10.13)^{*}$ \\
\hline \multirow{2}{*}{ Profession } & Midwives & 67 & 187 & $0.28(0.19-0.45)$ & $0.51(0.15-1.49)$ \\
\hline & Others & 76 & 61 & 1 & 1 \\
\hline \multirow{2}{*}{ Level of hospital } & General & 58 & 127 & 1 & 1 \\
\hline & Specialized/referral & 85 & 121 & $1.54(1.14-2.33)$ & $1.41(0.85-2.58)$ \\
\hline \multirow{3}{*}{ Qualification } & Lower level & 7 & 17 & 1 & 1 \\
\hline & Medium level & 55 & 158 & $0.85(0.33-2.15)$ & $2.3(0.69-7.78)$ \\
\hline & Higher level & 81 & 73 & $2.7(1.18-6.86)$ & $2.7(0.53-8.13)$ \\
\hline \multirow{3}{*}{ Anticipation of labor pain } & Mild pain & 17 & 42 & 1 & 1 \\
\hline & Moderate pain & 72 & 146 & $1.2(0.65-2.29)$ & $0.92(0.44-1.955)$ \\
\hline & Severe pain & 54 & 60 & $2.2(1.14-4.36)$ & $1.7(0.74-3.77)$ \\
\hline \multirow{2}{*}{ Knowledge } & Inadequate & 77 & 212 & 1 & 1 \\
\hline & Adequate & 66 & 36 & $5.05(3.12-8.18)$ & $2.7(1.37-5.23)^{*}$ \\
\hline \multirow{2}{*}{ Attitude } & Negative & 37 & 125 & 1 & 1 \\
\hline & Positive & 106 & 123 & $2.9(1.86-4.56)$ & $1.5(0.85-2.58)$ \\
\hline \multirow{2}{*}{ Availability of analgesics } & Not available & 42 & 151 & 1 & 1 \\
\hline & Available & 101 & 97 & $3.74(2.41-5.81)$ & $3.3(1.99-5.53)^{* *}$ \\
\hline \multirow{2}{*}{ Received training } & No & 75 & 156 & 1 & 1 \\
\hline & Yes & 68 & 92 & $1.54(1.13-2.33)$ & $1.13(0.68-1.87)$ \\
\hline
\end{tabular}

${ }^{*} P$ value $<0.05 .{ }^{* *} P$ value $\leq 0.001$. Lower level, diploma; Mid-level, BSc; Higher level, MSc, general practitioners, consultants, and residents; Others, medical doctors, anesthetists, and anesthesiologists. 
providing obstetric analgesics at least once in the last 12 months. Longer work experience, having adequate knowledge, and working in hospitals where analgesics were readily available were factors associated with utilization.

This finding is comparable with the study performed in Benin (38.9\%) [20]. But, it is lower than findings from Egypt $(70.1 \%)$ and Nigeria (49\% and 48.9\%) [15, 18, 21]. This variation might be due to differences in hospital protocols, availability of drugs and equipment, and availability of advanced health institutions and skilled health professionals. This could also be from sociocultural and socioeconomic variations. However, it is higher than the studies conducted in Bangladesh (7.4\%) and East Gojjam zone, Ethiopia (8.4\%) $[22,23]$. The variation might be due to differences in perception of labor pain and its management [12]. This could also arise from variation in the study period, sample size, and differences in the study setting.

Although its side effects are a concern, congruent with other findings in Nigeria and Gahanna, pethidine was the commonest analgesics utilized $[18,24]$. This may be because opioids are inexpensive and are simple to administer [25]. Regrettably, epidural analgesia was employed only by $12.6 \%$ of the participants. Although comparable with the findings from Egypt (12.4\%) [21], this is lower than findings from USA (58\%), Norway (25.9\%), and UK (25\%) [26-28]. This might be due to the limited number of skilled providers, unavailability of equipment, and lack of attention for labor pain management [17].

In line with findings from previous studies, providers with more years of experience were more likely to utilize labor analgesia $[23,29]$. This may be due to the reason that as obstetric care providers became more experienced, their level of knowledge and attitude became improved [30]. In addition, having adequate knowledge was associated with utilization of labor analgesia. This is similar with studies conducted in Nigeria and Ethiopia $[15,23]$. However, it is inconsistent with study performed in Kembata Tembaro Zone, Southern Ethiopia, which reported obstetric care providers with inadequate knowledge were practicing labor analgesia more likely [16]. This might be due to the differences in the supply of the analgesics which would be less in the peripheral settings compared to Addis Ababa where the pain-free labor initiative was initially launched and still underway. In settings where no supply exists, knowledge may not suffice for utilization. In addition, the variation in the study participants could result in variation. Our study included senior clinicians compared to the former study where majority of the participants were lowlevel cadres. This is supported by the study conducted in Australia showing that obstetricians prefer pharmacologic methods for labor pain relief [31]. Not surprisingly, availability of labor analgesics was significantly associated with utilization labor analgesics which is consistent with studies conducted in Ethiopia and Nigeria $[15,16]$.

\section{Conclusion}

Almost two in five obstetric care providers reported providing labor analgesia. Given the international practice and national direction toward pain-free labor, our findings are low. Providers with more years of work experience, having knowledge and working in hospitals where analgesics are readily available, were more likely to utilize labor analgesics. Ensuring adequate supply of analgesics and increasing awareness about importance of managing labor pain is essential to make sure that every woman would give birth without enduring labor pain, the most severe form of pain while giving life.

\section{Data Availability}

The data used to support the findings of this study are available from the corresponding author upon request.

\section{Conflicts of Interest}

The authors declare that they have no conflicts of interest.

\section{Authors' Contributions}

RG, TAY, and AKT conceived the study. RG analyzed the data and drafted the manuscript with continuous input from TA and AKT. TA and AKT supervise the overall work and reviewed the draft manuscript. AKT is the guarantor. All authors read and approved the final manuscript for submission.

\section{Acknowledgments}

The authors would like to thank Dilla University for funding the study. Their gratitude also go to respective hospital administrators, supervisors, data collectors, and the study participants.

\section{References}

[1] H. C. Lawrence, J. A. Copel, D. F. O'Keeffe et al., "Quality patient care in labor and delivery: a call to action," American Journal of Obstetrics and Gynecology, vol. 207, no. 3, pp. 147-148, 2012.

[2] ACOG, "ACOG practice bulletin no. 209: obstetric analgesia and anesthesia," Obstetrics Gynecology, vol. 133, no. 3, pp. e208-e225, 2019.

[3] L. Mårtensson and I. Bergh, "Effect of treatment for labor pain: verbal reports versus visual analogue scale scores-a prospective randomized study," International Journal of Nursing and Midwifery, vol. 3, no. 4, pp. 43-44, 2011.

[4] M. Tournaire and A. Theau-Yonneau, "Complementary and alternative approaches to pain relief during labor," EvidenceBased Complementary and Alternative Medicine, vol. 4, no. 4, pp. 409-417, 2007.

[5] S. Alehagen, B. Wijma, and K. Wijma, "Fear of childbirth before, during, and after childbirth," Acta Obstetricia et Gynecologica Scandinavica, vol. 85, no. 1, pp. 56-62, 2006.

[6] M. Aksoy, A. N. Aksoy, A. Dostbil, M. G. Celik, and I. Ince, "The relationship between fear of childbirth and women's knowledge about painless childbirth," Obstetrics and Gynecology International, vol. 2014, Article ID 274303, 7 pages, 2014.

[7] Centers for Disease Control and Prevention, Morbidity and Mortality Weekly Report, vol. 66, no. 6, pp. 30329-34027, Centers for Disease Control and Prevention, Atlanta, GA, 
USA, 2017, https://wwwhealthypeoplegov/2020/topicsobjectives/topic/maternal-infantand-child-health/objectives.

[8] T. Melese, Y. Gebrehiwot, and D. Habte, "Assessment of client satisfaction in labor and deliver services at maternity referral hospitals in Ethiopia," The Pan African Medical Journal, vol. 17, no. 7, p. 76, 2014.

[9] Y. Tebekaw, Y. Mashalla, and G. Thupayagale, "Patterns of cesarean delivery in Addis Ababa,Ethiopia," African Journal of Primary Health Care and Family Medicine, vol. 8, no. 2, pp. 106-111, 2014.

[10] J. A. Crowhurst, Dewhurst's Text Book of Obstetrics Gynecology, Blackwell Publishing, Malden, MA, USA, 7th edition, 2007.

[11] M. J. Osterman and J. A. Martin, "Epidural and spinal anesthesia use during labor: 27-state reporting area," National Vital Statistics Reports, vol. 59, no. 16, pp. 1-13, 2011.

[12] K. Shammi, H. Yu, K. Sourabh, L. Chen, and D. Qiao, "Women's awareness and attitudes towards labor analgesia influencing practice between developed and developing countries," Advances in Reproductive Sciences, vol. 4, no. 2, pp. 46-52, 2016.

[13] EFMHACA, "Standard treatment guidelines for general hospital: good prescribing and dispensing practices for better health outcomes," 2014, http://wwwpascar.org.

[14] McC. Mary, C. Stewart, and K. Birhanu, "A survey of healthcare providers' knowledge and attitudes regarding pain relief in labor for women in Ethiopia," BMC Pregnancy and Childbirth, vol. 17, no. 1, p. 56, 2017.

[15] E. Ogboli-Nwasor, S. E. Adaji, S. B. Bature, and O. S. Shittu, "Pain relief in labor: a survey of awareness, attitude, and practice of health care providers in Zaria, Nigeria," Journal of Pain Research, vol. 2011, no. 4, p. 227, 2011.

[16] T. E. Geltore, A. Taye, and A. G. Kelbore, "Utilization of obstetric analgesia in labor pain management and associated factors among obstetric caregivers in public health facilities of Kembata Tembaro Zone, Southern Ethiopia," Journal of Pain Research, vol. 11, no. 3, pp. 3089-3097, 2018.

[17] A. Bitew, A. Workie, T. Seyum, and T. Demeke, "Utilization of obstetric analgesia in labor pain management and associated factors among obstetric care givers in Amhara Regional State Referral Hospitals, Northwest Ethiopia. A hospital based cross sectional study," Journal of Biomedical Sciences, vol. 5, no. 2, 2016.

[18] L. O. Lawani, J. N. Eze, O. B. Anozie, C. A. Iyoke, and N. N. Ekem, "Obstetric analgesia for vaginal birth in contemporary obstetrics: a survey of the practice of obstetricians in Nigeria," BMC Pregnancy and Childbirth, vol. 14, no. 1, p. 140, 2014.

[19] [The Helsinki Declaration of the World Medical Association (WMA), "Ethical principles of medical research involving human subjects]," Polski Merkuriusz Lekarski, vol. 36, no. 215, pp. 298-301, 2014.

[20] C. O. Imarengiaye, "Trends in pain relief in labor: implications for obstetric analgesia services in Nigeria," Postgrad Medicine Journal, vol. 12, no. 3, pp. 193-202, 2006.

[21] O. Mousa, A. A. Abdelhafez, A. R. Abdelraheim, A. M. Yousef, A. A. Ghaney, and S. El Gelany, "Perceptions and practice of labor pain-relief methods among health professionals conducting delivery in minia maternity units in Egypt," Obstetrics and Gynecology International, vol. 2018, Article ID 3060953, 6 pages, 2018.

[22] S. Tasnim, "Perception about pain relief during normal labour among health care providers conducting delivery," Medicine Today, vol. 22, no. 1, pp. 20-23, 2010.
[23] K. Anteneh Bishaw, E. Gemechu Sendo, and W. Sinishaw Abebe, "Knowledge, and use of labor pain relief methods and associated factors among obstetric caregivers at public health centers of East Gojjam zone, Amhara region, Ethiopia," BMC Pregnancy and Childbirth, vol. 20, no. 180, p. 6, 2020.

[24] L. Aziato, A. A. Kyei, and G. Deku, "Experiences of midwives on pharmacological and non-pharmacological labour pain management in Ghana," Reproductive Health, vol. 14, no. 1, p. 128, 2017.

[25] M. Heesen and M. Klimek, "Obstetric analgesia-update 2016," Journal of Perinatal Medicine, vol. 45, no. 3, pp. 281-289, 2017.

[26] D. Eugene, C. Sakala, and S. Applebaum, "Listening to mothers II: report of the second national US survey of women's childbearing experiences," The Journal of Perinatal Education, vol. 16, no. 4, p. 9, 2007.

[27] T. O. Tveit, A. Halvorsen, and J. H. Rosland, "Analgesia for labour: a survey of Norwegian practice-with a focus on parenteral opioids," Acta Obstetricia et Gynecologica Scandinavica, vol. 53, no. 9, pp. 794-799, 2009.

[28] R. Baraz and R. E. Collis, "The management of accidental dural puncture during labour epidural analgesia: a survey of UK practice," Anaesthesia, vol. 60, no. 7, pp. 673-679, 2005.

[29] B. Ruschel Hillmann and A. Maria Nunes de Faria Stamm, "Knowledge, attitude and practice regarding pharmacological methods of labor analgesia," Brazil Joural Publicaton São Paulo, vol. 2, no. 1, pp. 14-19, 2019.

[30] A. P. Aschenbrenner, L. Hanson, T. S. Johnson, and S. T. Kelber, "Nurses' own birth experiences influence labor support attitudes and behaviors," Journal of Obstetric, Gynecologic \& Neonatal Nursing, vol. 45, no. 4, pp. 491-501, 2016.

[31] K. L. Madden, D. Turnbull, A. M. Cyna, P. Adelson, and C. Wilkinson, "Pain relief for childbirth: the preferences of pregnant women, midwives and obstetricians," Women and Birth, vol. 26, no. 1, pp. 33-40, 2013. 